\title{
"Some people see innovation as change, but we have never really seen it like that. It's understanding things and making them better." Adapted from Tim Cook, Chief Executive Officer of Apple
}

\author{
Justin Van Meeteren, DO, and Simon Maltais, MD, PhD
}

\author{
From Cardiac Surgery, Mayo Clinic, Rochester, Minn. \\ Disclosures: Authors have nothing to disclose with regard to commercial support. \\ Received for publication March 21, 2016; accepted for publication March 24, 2016; available ahead of print May \\ $11,2016$. \\ Address for reprints: Simon Maltais, MD, PhD, Cardiac Surgery, Mayo Clinic, 200 First St SW, Rochester, MN \\ 55905 (E-mail: maltais.simon@mayo.edu). \\ J Thorac Cardiovasc Surg 2016;152:628-9 \\ $0022-5223 / \$ 36.00$ \\ Copyright (c) 2016 Published by Elsevier Inc. on behalf of The American Association for Thoracic Surgery \\ http://dx.doi.org/10.1016/j.jtcvs.2016.03.057
}

In this issue of the Journal, Goel and colleagues ${ }^{1}$ discuss the natural history and treatment of a case of apical variant of hypertrophic cardiomyopathy (ApHCM). The authors illustrate the symptoms associated with the progression of the disease and detail a novel surgical treatment for this pathology. After successful transaneurysmal midventricular ventriculotomy, the authors discuss the pathophysiology of

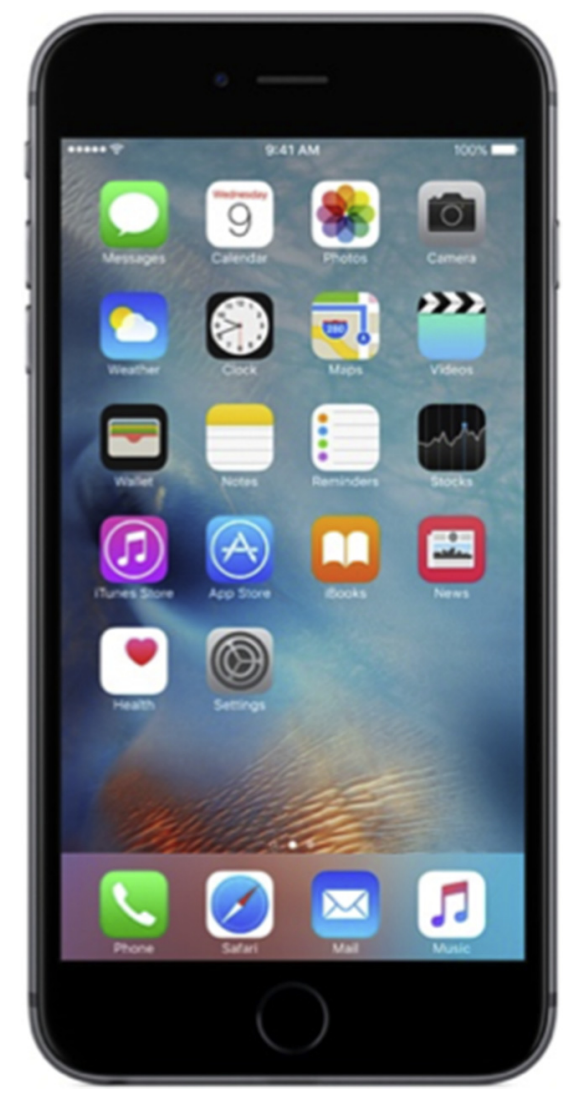

FIGURE 1. A better strategy for addressing patients with ApHCM.

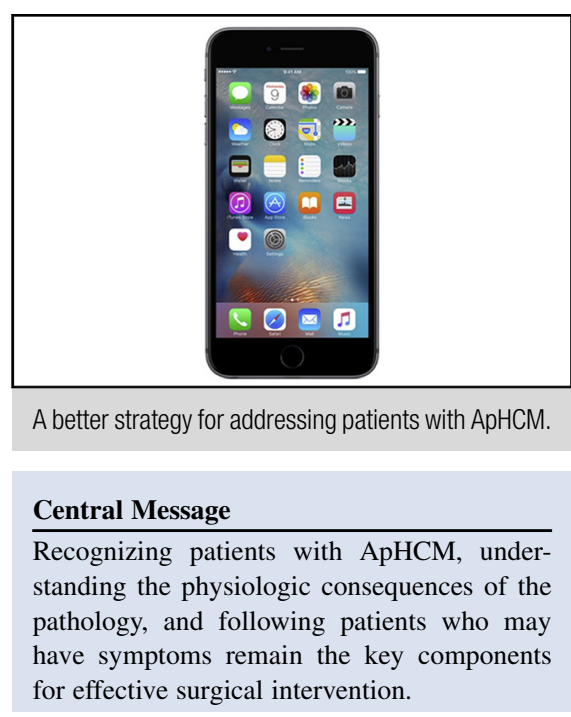

See Article page 626

the disease and the potential benefits of the procedure in enhancing ventricular diastolic filling, improving symptoms, and preventing complications. This case helps our understanding of the disease and provides insight into the complex approach to this pathology. It allows our community to recognize that this challenging problem could be addressed using conventional surgical techniques before referring patients for heart replacement therapies.

\section{LEARNING FROM THE PAST}

Our institution was among the first to describe the midventricular transapical myectomy. ApHCM generally is not associated with subaortic obstruction, and symptoms (when present) respond poorly to medical treatment. The transapical approach for ApHCM involves removal of hypertrophied muscle in the apical and middle regions of the left ventricle (LV), thus creating a larger LV chamber size in diastole. The LV contractility generally is preserved, resulting in improved effective stroke volume with reduced end-diastolic pressures. Because this disease entity is rare and poorly understood, patients often are referred for heart transplantation and remain poor candidates for durable assist devices because of severe LV hypertrophy. 


\section{A SPECIALIZED SURGICAL INTERVENTION}

Surgical treatment of patients with obstructive hypertrophic cardiomyopathy (HCM) involves subaortic myectomy to relieve outflow tract obstruction present in more than $70 \%$ of patients. Medical treatment also is effective in reducing dynamic outflow obstruction and symptoms. These procedures commonly are diagnosed and performed in centers of excellence where HCM is approached as a multidisciplinary team. Recognizing patients with ApHCM, understanding the physiologic consequences of the pathology, and following patients who may develop symptoms remain key components to early and effective surgical intervention. Removal of an adequate volume of ventricular muscle is challenging and associated with potential injury to papillary muscles (displaced apically). A small ventriculotomy should be made first to identify the anatomic position of papillary muscles, and as the ventriculotomy proceeds, these muscles can be shaved to ensure a maximized LV volume with decreased risks of residual midventricular obstruction. The art of the surgical intervention relies on appropriate patient selection, including careful assessment of LV end-diastolic volumes. Although LV morphology makes imaging challenging, experienced HCM teams help carry the indication and ensure adequate treatment of the condition. Recognizing the presence and pathophysiology of the apical aneurysm is critical for surgical planning.

\section{WE "OWE" IT TO OUR PATIENTS}

Although the current report by Goel and colleagues ${ }^{1}$ demonstrates a case of ApHCM illustrating the natural history and successful treatment of this condition, it details the importance of a collaborative strategy. As advanced heart failure therapies can be considered, we must reserve these strategies after a thorough evaluation in specialized HCM centers, where benchmarks have been set through the years for a conventional surgical approach of this complex disease entity (Figure 1).

\section{Reference}

1. Goel K, Schaff HV, Nishimura RA. Natural history of apical hypertrophic cardiomyopathy and novel surgical treatment. J Thorac Cardiovasc Surg. 2016;152: 626-7. 